\title{
MiR-31a-5p protects myocardial cells against apoptosis by targeting $\mathrm{Tp53}$
}

\author{
MENG-JIE YAN ${ }^{1}$, ZHI-SEN TIAN ${ }^{2}$, ZHI-HUI ZHAO ${ }^{3}$ and PING YANG ${ }^{1}$ \\ Departments of ${ }^{1}$ Internal Medicine and Cardiology, and ${ }^{2}$ Orthopedics, China-Japan Union Hospital of Jilin University; \\ ${ }^{3}$ College of Animal Science and Veterinary Medicine, Jilin University, Changchun, Jilin 130033, P.R. China
}

Received March 22, 2017; Accepted September 11, 2017

DOI: $10.3892 / \mathrm{mmr} .2017 .8357$

\begin{abstract}
The pathogenesis and progression of heart failure (HF) involves multiple mechanisms, including the increased activity of the renin-angiotensin-aldosterone system, apoptosis and differential expression of microRNAs (miRNAs/miRs). Our previous study revealed an increase in miR-31a-5p levels in the failing hearts of a rat HF model. In the present study, whether and how miR-31a-5p mediates angiotensin II (AngII)-induced apoptosis in the cardiac H9C2 cell line, was investigated using molecular biological approaches, including reverse transcription followed by quantitative polymerase chain reaction, western blotting, RNA arrays, and mutagenesis. It was demonstrated that AngII stimulation increased apoptosis and decreased miR-31a-5p expression, which coincided with increased tumor protein p53 (Tp53) levels. Overexpression of miR-31a-5p significantly suppressed the AngII-induced apoptotic rate and caspase- 3 activity, while suppression of miR-31a-5p did the opposite. A total of 16 proapoptotic genes that were downregulated and 4 antiapoptotic genes that were upregulated in the miR-31a-5p-overexpressed cells were identified. It was also revealed that Tp53 mRNA contained the seed sequence in its 3'-untranslated region for miR-31a-5p binding. The luciferase reporter analysis showed that miR-31a-5p repressed the luciferase activity of the wild-type seed sequence, but not the mutated seed sequence fused to a reporter construct. Thus, it was demonstrated that miR-31a-5p mediated AngII-triggered apoptosis in myocardial cells at least partially through targeting Tp53. These findings advance the understanding of the functional interaction between miRNAs and Tp53 in the setting of cardiac diseases. Further work is required to explore whether miR-31a-5p can serve as a therapeutic target for HF treatment in vivo.
\end{abstract}

Correspondence to: Dr Ping Yang, Department of Internal Medicine and Cardiology, China-Japan Union Hospital of Jilin University, 126 Xiantai Road, Changchun, Jilin 130033, P.R. China E-mail: pyang@jlu.edu.cn

Key words: miR-31a-5p, apoptosis, Tp53

\section{Introduction}

Apoptosis, also known as programmed cell death, plays important roles in a variety of cellular events in both physiological and pathological settings and disease development $(1,2)$, including the pathogenesis of heart diseases such as myocardial infarction (MI) and heart failure (HF) $(3,4)$, as evidenced by the findings that abnormal apoptosis of myocytes promotes the development of HF, arrhythmogenic right ventricular dysplasia, and dilated cardiomyopathy (2). Moreover, very low levels of cardiac myocyte apoptosis were found to be sufficient to cause cardiomyopathy and HF, and correspondingly, the inhibition of myocyte apoptosis prevented the development of cardiomyopathy (5). Therefore, targeting apoptosis is a potential avenue for the clinical treatment of certain cardiac diseases such as HF.

It has been well documented that the renin-angiotensin-aldosterone system (RAAS) is activated in and contributes to the pathogenesis of a number of cardiovascular diseases, including HF and MI (6). Angiotensin II (AngII), as an important component of the RAAS, is also increased in these cardiac disease settings and induces cardiomyocyte apoptosis (7). Therefore, angiotensin-converting enzyme inhibitors (ACEIs) have been used to treat patients with HF and MI in the clinic (8-11). Although it is well known that AngII induces cardiomyocyte apoptosis via at least two mechanisms: Oxidative stress and DNA damage $(7,12)$, the exact mechanisms linking AngII stimulation and pathological changes remain uncertain, and the complete network that mediates the cellular responses to AngII treatment remains to be deciphered.

MicroRNAs (miRNAs) are a class of short, noncoding RNAs that downregulate the expression of target genes by binding to the $3^{\prime}$-untranslated region ( $3^{\prime} \mathrm{UTR}$ ). Numerous studies have reported dysregulation and involvement of miRNA expression in pathological states including cardiovascular disorders (13). For instance, miR-21 protects myocytes against $\mathrm{H}_{2} \mathrm{O}_{2}$-induced injury by targeting $\mathrm{PDCD}_{4}(14)$, and miR-214 promotes cell survival and suppresses cell apoptosis $(15,16)$. In addition, miR-31 inhibits tumor metastasis though blocking the cell cycle and inducing apoptosis, and it is regarded as a tumor suppressor-related miRNA (17). The role of miRNAs in the RAAS system is just emerging (18). For example, miR-155, which plays an important role in the pathogenesis of cardiac diseases (19), serves as a negative regulator of AngII-promoted 
cell proliferation via targeting AT1R (20), while overexpression of miR-132 and miR-212 potentiates AngII activity and mediates the expression of a number of genes involved in AngII signaling $(21,22)$. However, whether other miRNAs and novel mechanisms are involved in AngII-mediated apoptosis remains to be elucidated.

MiR-31a-5p is the leading member of miR-31 family, but limited studies have been performed to investigate its roles in physiological and pathophysiological events. A previous study has shown that MiR-31a-5p is linked to colorectal cancer (23). Our more recent work revealed that the miR-31a-5p level was significantly higher in the hearts of rats with post-infarction HF than that in the sham group (24). However, the exact role of miR-31a-5p in the pathogenesis of HF remains uncertain. Given the increased expression of miR-31a-5p in failing hearts, we hypothesized that miR-31a-5p may play a potential role in the development of HF. In the present study, we investigated whether miR-31a-5p mediates myocardial apoptosis in $\mathrm{H} 9 \mathrm{C} 2$ cells, a cardiac cell line, in response to AngII stimulation.

\section{Materials and methods}

Cell culture. H9C2 cells were obtained from the Center Laboratory of China-Japan Union Hospital. Cells were cultured in Dulbecco's modified Eagle medium with Nutrient Mixture F-12 (DMEM/F12) containing 10\% fetal bovine serum and $1 \%$ antibiotics in a modular incubator with an atmosphere of $5 \% \mathrm{CO}_{2} / 95 \%$ air at $37^{\circ} \mathrm{C}$. When cells reached $70-80 \%$ confluence, they were seeded in 6-well and 96-well plates.

AngII stimulation and transfection. H9C2 cells in 6-well plates were transfected with FAM fluorescently labeled miRNA mimics, inhibitor, and negative control (NC; GenePharma, Shanghai, China), respectively, with Fugene HD (Promega Corp., Madison, WI, USA). The transfection was performed in two groups: Vehicle and AngII groups. Experiments were executed in three independent assays, with each carried out in duplicate. Transfection efficiency was evaluated by a fluorescence microscope at 6 to $24 \mathrm{~h}$ after transfection. AngII $\left(10^{-9} \mathrm{M}\right)$ was added to cells of the AngII group; this concentration has been reported to present a maximal apoptotic rate in myocytes (25).

Flow cytometry. After transfection, H9C2 cells in different groups were digested by EDTA-free trypsin, washed twice with phosphate-buffered saline (PBS), and then resuspended in $500 \mu \mathrm{l}$ of $1 \mathrm{x}$ binding buffer. Harvested cells were incubated with $5 \mu \mathrm{l}$ of Annexin $\mathrm{V}$ fluorescein isothiocyanate and $5 \mu \mathrm{l}$ of propidium iodide (Annexin V:FITC Apoptosis Detection kit I; BD Biosciences, San Diego, CA, USA) for $20 \mathrm{~min}$ at room temperature. Thereafter, the cell suspensions were analyzed by flow cytometry (BD LSRFortessa X-20; BD Biosciences). The data were analyzed using FlowJo 7.61 software (Treestar Inc., Ashland, OR, USA).

Total RNA extraction and reverse transcription $(R T)$. Total RNA of transfected cells was extracted using TRIzol reagent (Takara Bio, Inc., Otsu, Japan), and the RNA concentration and integrity were evaluated by a NanoDrop 2000 spectrophotometer (Thermo Fisher Scientific Inc., Wilmington, DE, USA) and agarose gel electrophoresis. A total of $1 \mu \mathrm{g}$ of purified RNA was used for reverse transcription using an RT kit (Takara Bio, Inc.), according to the manufacturer's protocol. RT primers for miR-31a-5p were designed using the stem ring method. cDNA was stored at $-80^{\circ} \mathrm{C}$.

Prediction of target genes and primer design. The target genes for miR-31a-5p were predicted using bioinformatics tools. The specific primers for these genes were designed by Primer 5.0. The selected genes were further verified by real-time PCR. A $20-\mu 1$ system consisting of $2 \mu \mathrm{l}$ of cDNA, $0.4 \mu \mathrm{l}$ of forward/reverse primers, $10 \mu \mathrm{l}$ of SYBR-Green qPCR Master Mix (Takara Bio, Inc.), and $7.2 \mu \mathrm{l}$ of RNase-free water was run on an Mx3005P (Agilent Technologies, Inc., Santa Clara, CA, USA). The results were analyzed using the $2^{-\Delta \Delta \mathrm{CT}}$ method. The data were analyzed using SPSS Statistics 19 software (IBM Corp., Armonk, NY, USA).

Total protein extraction and western blot. Total protein was extracted from H9C2 cells using RIPA protein lysis buffer (Beyotime Institute of Biotechnology, Haimen, China) with $1 \%$ protease inhibitors (Bestbio, Shanghai, China). Briefly, cells in different groups were harvested using trypsin, washed with cold PBS twice, incubated with $100 \mu \mathrm{l}$ of lysis buffer on ice for $30 \mathrm{~min}$, and centrifuged at 12,000 rpm for 18-30 min. The concentration of protein products was monitored using a BCA kit (KenGen BioTECH, Nanjing, China), according to the manufacturer's protocol. Supernatant protein products were stored at $-80^{\circ} \mathrm{C}$.

For western blot, briefly, $25 \mu \mathrm{g}$ of protein samples from different transfection groups were resolved by sodium dodecyl sulfate-polyacrylamide gel electrophoresis and transferred to polyvinylidenefluoride membranes. Membranes were probed with rabbit polyclonal anti-rat tumor protein p53 (Tp53) and rabbit monoclonal anti- $\beta$-actin primary antibodies (Abcam, USA) at $4^{\circ} \mathrm{C}$ overnight. Then, the membranes were washed with tris-buffered saline containing Tween-20 and further incubated with the appropriate horseradish peroxidase-conjugated secondary antibody (Abcam, Cambridge, MA, USA) at room temperature for $1.5 \mathrm{~h}$. The protein bands were detected by an ImageQuant LAS 4000 Mini instrument (GE Healthcare Bio-Sciences AB, Uppsala, Sweden) and a SuperSignal West Pico Chemiluminescence kit (Thermo Fisher Scientific Inc., Waltham, MA, USA). The protein bands were quantified by ImageJ software (National Institutes of Health, Bethesda, MD, USA).

Caspase-3 activity determination. Caspase-3 activity was determined using a Caspase-3 Activity Assay kit (Bestbio), according to the manufacturer's protocol. Briefly, $50 \mu \mathrm{g}$ of protein sample was added to $90 \mu \mathrm{l}$ of detection buffer and $10 \mu \mathrm{l}$ of Ac-DEVD-pNA, and the mixture was incubated at $37^{\circ} \mathrm{C}$ for $1 \mathrm{~h}$. Next, the absorbance at $405 \mathrm{~nm}$ was measured. The ratio of the reading from the experimental group to that of the control group was the relative caspase-3 activity.

Rat apoptosis $R^{2}$ Profiler $^{T M}$ PCR Array analysis. The Rat Apoptosis RT2 Profiler $^{\mathrm{TM}}$ PCR Array (Qiagen, GmbH, Hilden, Germany) contains 84 apoptotic pathway-related genes. Total 

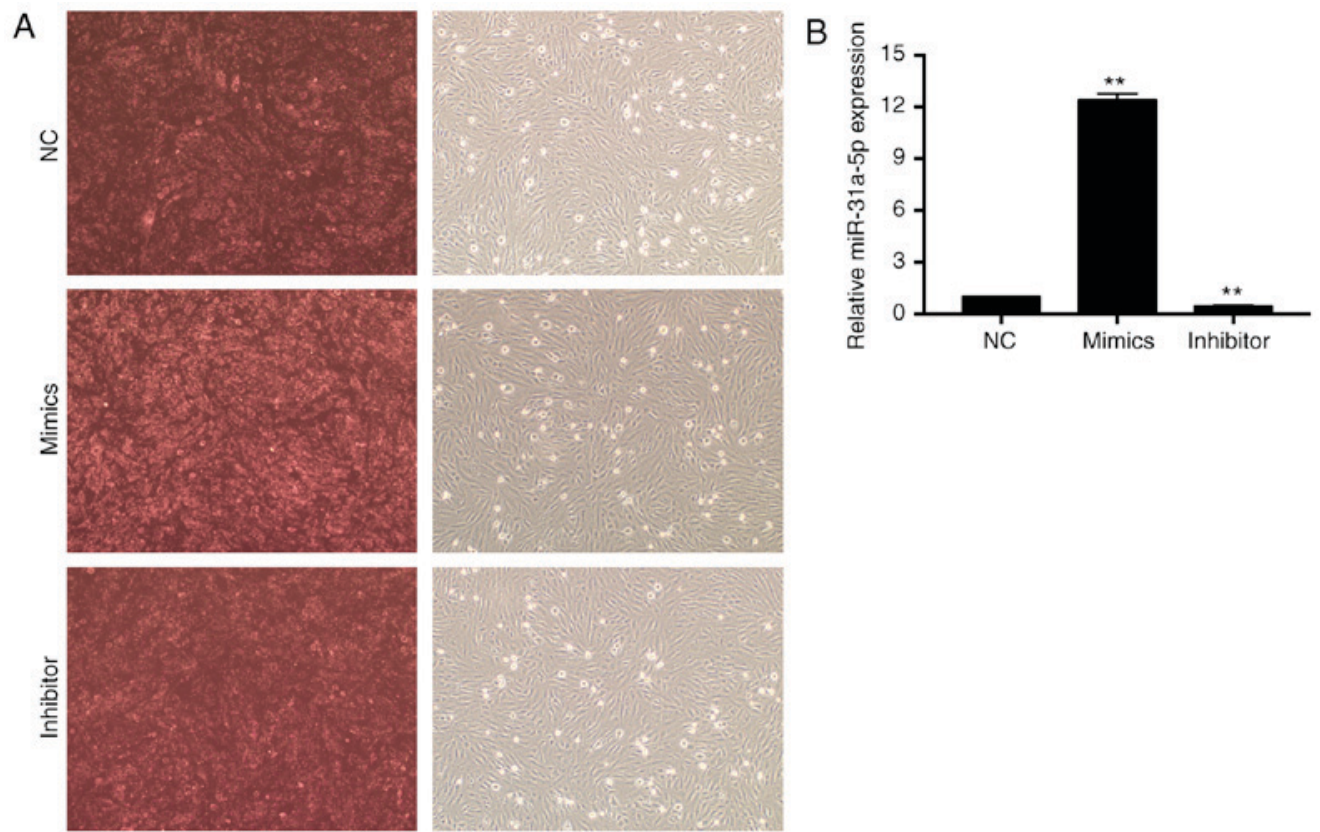

Figure 1. Evaluation of the transfection efficiency of miR-31a-5p mimics. (A) H9C2 cells were stained red by FAM-labeled miR mimics, inhibitor, and NC. (B) Evaluation of miR-31a-5p expression in H9C2 cells transfected with mimics, inhibitor, and NC by RT-qPCR. ${ }^{* *} \mathrm{P}<0.01$ vs. NC. miR, microRNA; NC, negative control.

RNA was extracted using an RNA extraction kit (Qiagen $\mathrm{GmbH}$, Hilden, Germany), according to the manufacturer's protocol. RT reactions were performed using $1 \mathrm{ng}$ of RNA and an RT kit (Qiagen). Housekeeping genes such as $\beta$-actin and $\beta-2$ microglobulin were used for normalization. The values of controls in the plate were used to determine the expression of each target gene present in the plate. Equal aliquots of cDNA and $\mathrm{RT}^{2}$ SYBR Green qPCR Master Mix (Qiagen) were added to each well of the same PCR Array plate containing the gene-specific primer sets. The data with the threshold values were exported and analyzed using the RT ${ }^{2}$ Profiler PCR Array Data Analysis template v. 3.3 (SuperArray Biosciences; Qiagen).

Luciferase reporter assay. To verify that $\mathrm{Tp} 53$ is a direct target for miR-31a-5p, we constructed a target reporter using the pmiR-RB-REPORT ${ }^{\mathrm{TM}}$ carrier (Ribobio, Guangzhou, China). An approximately 200-bp fragment of the 3'UTR of Tp53 mRNA was synthesized (Genewiz, Inc., South Plainfield, NJ, USA), which carried two restriction sites, Not I $\left(\mathrm{GC}^{\wedge} \mathrm{GGCCGC}\right)$ and $X h o \mathrm{I}\left(\mathrm{C}^{\wedge} \mathrm{TCGAG}\right)$. This fragment was then inserted into the vector pmiR-RB-REPORT ${ }^{\mathrm{TM}}$ (designed as pmiR-RB-REPORT ${ }^{\mathrm{TM}}-\mathrm{WT}$ ) on these two sites. Targeted mutagenesis was performed to generate the desired mutations on the potential miR-31a-5p target site (from WT, TCCTTTCTTGCC ATTTTA, to mutant TCCTTCATGTAGCATTTTA), designed as pmiR-RB-REPORT ${ }^{\mathrm{TM}}$-mut. H9C2 cells seeded in 24-well plates were cotransfected with pmiR-RB-REPORT ${ }^{\mathrm{TM}}-\mathrm{WT}$ or pmiR-RB-REPORT ${ }^{\mathrm{TM}}$-mut together with miR-31a-5p mimics or NC duplex (GenePharma) using a FuGene HD transfection reagent. pmiR-RB-REPORT ${ }^{\mathrm{TM}}$ was transfected as a control. After $48 \mathrm{~h}$, the cells were harvested, and the luciferase activity was measured using a dual-luciferase reporter assay kit (Promega Corp.) and recorded with a multi-plate reader (Synergy 2; BioTek Instruments, Inc., Winooski, VT, USA).
Statistical analysis. Data are presented as the mean \pm standard deviation and were compared using a two-tailed t-test for two groups or one-way analysis of variance for multiple groups. Correlation analysis was performed using Spearman's correlation coefficient. $\mathrm{P}<0.05$ was considered to indicate a statistically significant difference.

\section{Results}

MiR-31a-5p attenuated AngII-induced apoptosis. The transfection efficiency of FAM-labeled siRNAs was first evaluated by fluorescence microscopy. As shown in Fig. 1A, 80-90\% of cells were stained red. Next, real-time PCR was performed to examine the expression levels of transfected miR-31a-5p. As shown in Fig. 1B, the expression level of miR-31a-5p in the group transfected with mimics was significantly higher than that in the control group $(\mathrm{P}<0.01)$, while the miR-31a-5p level in the inhibitor group was greatly suppressed compared with the $\mathrm{NC}$ group $(\mathrm{P}<0.01)$.

Next, we evaluated cell apoptosis in different groups with fluorescence correlation microscopy. Overexpression of miR-31a-5p decreased the apoptotic rate induced by AngII, while miR-31a-5p only slightly decreased cell apoptosis in the absence of AngII (Fig. 2A). We also measured the caspase-3 activity, and the results were consistent with those obtained by fluorescence correlation microscopy (Fig. 2B). Therefore, we concluded that miR-31a-5p protects myocytes against AngII-induced apoptosis.

Next, we measured the expression of apoptosis-related genes by $\mathrm{RT}^{2}$ Profiler ${ }^{\mathrm{TM}}$ PCR Array analysis and found that 20 apoptosis-related genes exhibited differential expression in the mimic-transfected group compared to the control group, among which 16 proapoptotic genes were downregulated and 4 antiapoptotic genes were upregulated (Fig. 2C). 

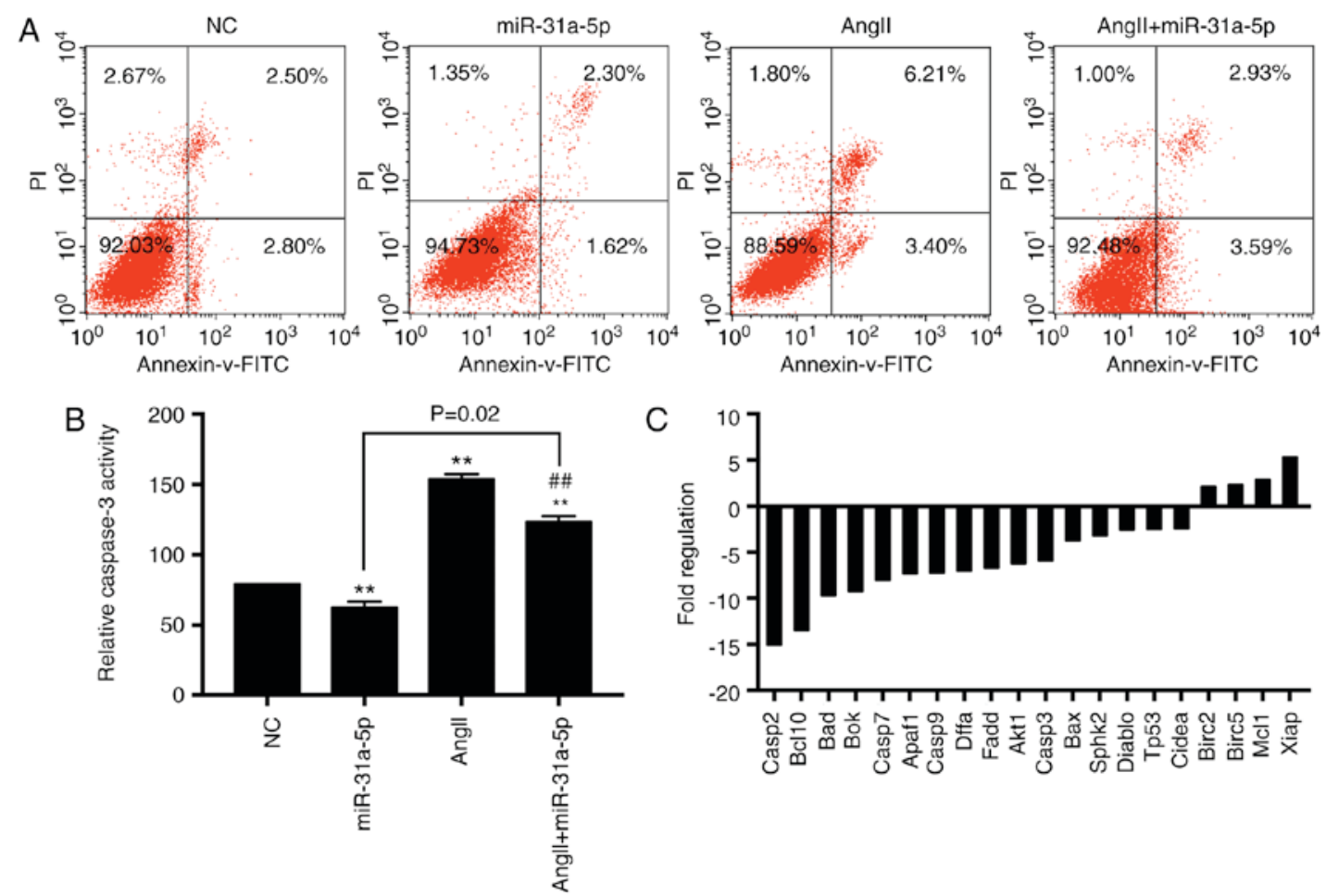

Figure 2. MiR-31a-5p protects H9C2 cells from apoptosis. (A) Flow cytometry was performed to identify apoptotic cells (red) stained with anti-Annexin V and propidium iodide. The percentage of apoptotic cells is indicated in each corner of each panel. Early apoptotic cells are shown in the lower right quadrant and late apoptotic cells in the upper right quadrant. (B) A caspase-3 activity assay kit was used to evaluate the caspase-3 activity in cells of microRNA mimic-, inhibitor-, and NC-transfected groups. ${ }^{* *} \mathrm{P}<0.01$ vs. NC, ${ }^{\# \#} \mathrm{P}<0.01$ vs. the AngII group. (C) Rat Apoptosis RT ${ }^{2}$ Profiler $^{\mathrm{TM}}$ PCR Array analysis revealed that 16 proapoptotic genes were decreased and 4 antiapoptotic genes were increased significantly in miR-31a-5p-overexpressed cells. NC, negative control; AngII, angiotensin II; miR, microRNA.
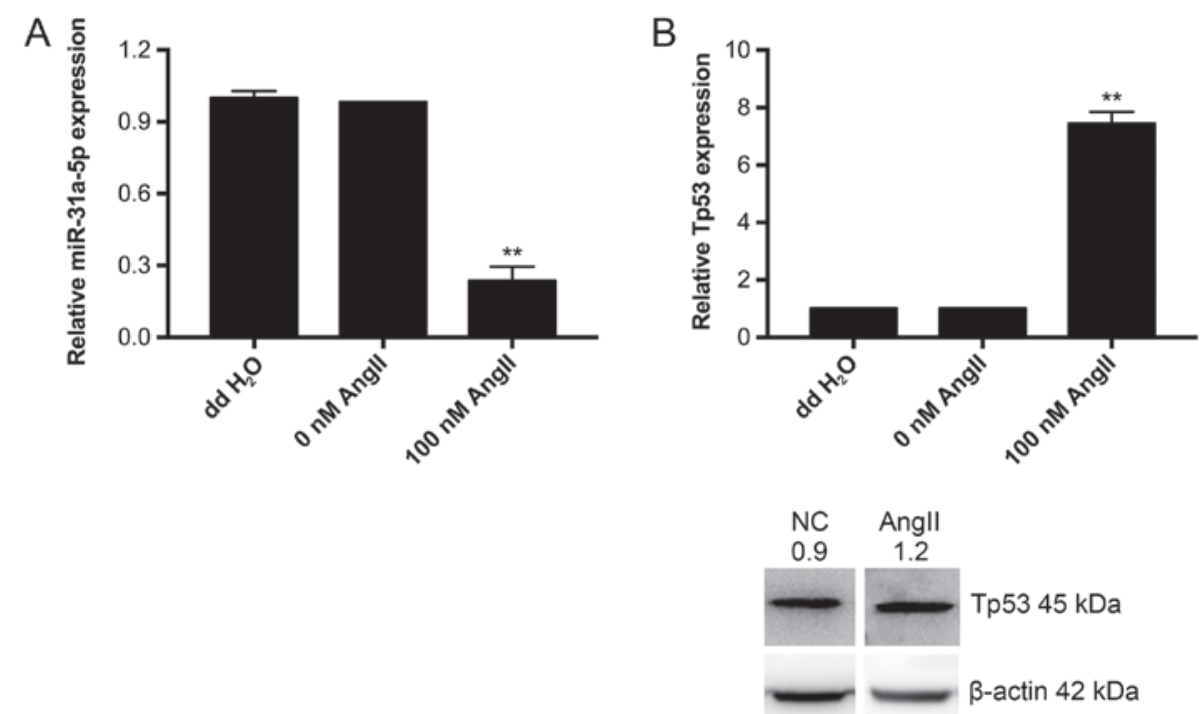

Figure 3. AngII treatment decreased the expression of miR-31a-5p but increased the expression of Tp53. Evaluation of miR-31a-5p and Tp53 levels by RT-qPCR (A) and western blotting (B). $\beta$-Actin was used as a loading control. ${ }^{* *} \mathrm{P}<0.01$ vs. vehicle ( 0 group). $\mathrm{N}=3$ per group. AngII, angiotensin II; miR, microRNA; $\mathrm{Tp} 53$, tumor protein $\mathrm{p} 53$.

AngII decreases the expression of miR-31a-5p and increases the expression of Tp53. After stimulation with $10^{-9} \mathrm{M}$ AngII for $24 \mathrm{~h}$, we measured the expression of miR-31a-5p and Tp53 by real-time PCR and western blot. The results showed a significant decrease in miR-31a-5p expression (Fig. 3A) and a substantial increase in $\mathrm{Tp} 53$ expression (Fig. 3B).

\section{Tp53 5' 5 ...CCCCGUCCCCUCCUUUCUUGCCA... miR-31-5p 3' GUCGAUACGGUCGUAGAACGGA}

Figure 4. miR-31a-5p showed complementarily base paired with the $3^{\prime}$ UTR region of Tp53. miR, microRNA; 3'UTR, 3'-untranslated region tumor; Tp53, protein $\mathrm{p} 53$. 


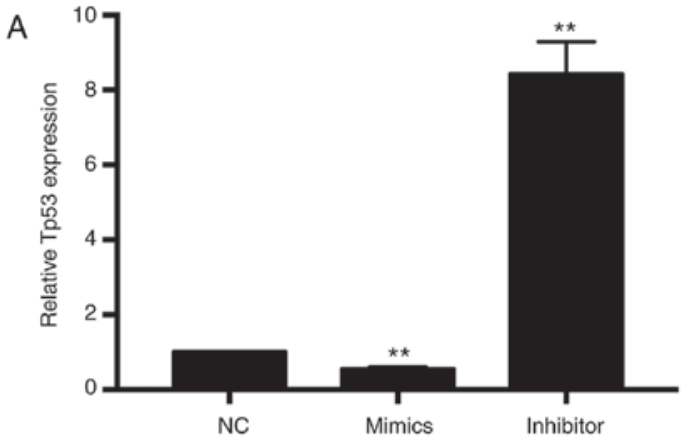

B
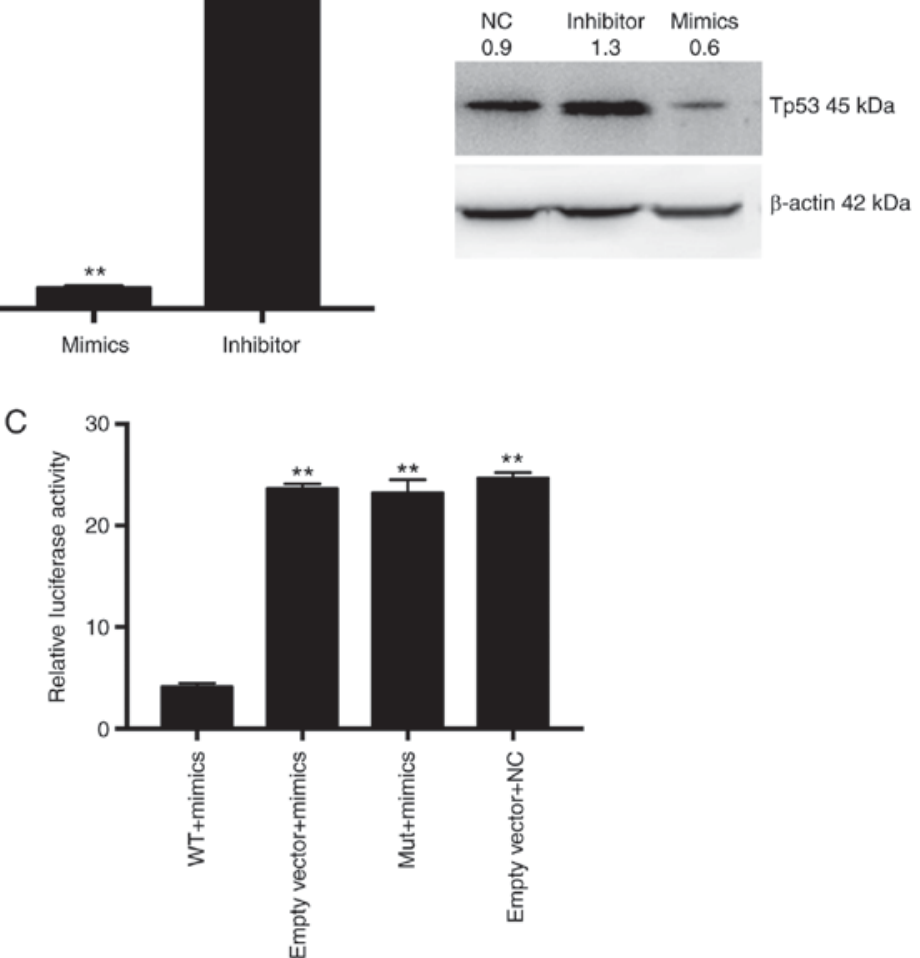

Figure 5. Tp53 was identified as a direct target of miR-31a-5p. Tp53 expression was mediated by the miR-31a-5p levels in H9C2 cells transfected with mimics, inhibitor, and NC as evaluated by (A) RT-qPCR and (B) western blot analyses. ${ }^{* *} \mathrm{P}<0.01$ vs. NC. $\beta$-Actin was used as an internal control. (C) miR-31a-5p targeted the 3'UTR of Tp53. Reporter assays were carried out as described in the materials and methods. Data were compiled from three independent experiments. NC, negative control; miR, microRNA; 3'UTR, 3'-untranslated region tumor; Tp53, protein p53.

Hence, we conclude that miR-31a-5p and Tp53 participate in AngII-induced apoptosis.

Tp53 is a direct target of miR-31a-5p. We screened several genes involved in cell cycle progression and apoptosis. tumor protein 53 (Tp53) was one of them. We also found that miR-31a-5p complementarily base paired with the $3^{\prime}$ UTR region of Tp 53 mRNA (Fig. 4). Moreover, Tp53 was shown to be significantly downregulated in an HF animal model in our previous study (unpublished). Therefore, Tp53 was chosen for further investigation. We first examined the Tp53 expression level in different transfection groups using real-time PCR (Fig. 5A) and western blot (Fig. 5B). Overexpression of miR-31a-5p inhibited the Tp53 expression level, while the miR-31a-5p inhibitor increased the Tp53 level. Since miRNAs bind to the seed sequence in the $3^{\prime}$ UTR of target genes to cause degradation or decrease the expression level of target genes, we subcloned the wild-type and mutated target sequence of miR-31a-5p located on the 3'UTR of Tp53 into pmiR-RB-REPORT ${ }^{\mathrm{TM}}$ to explore whether miR-31a-5p could mediate their activity. As shown in Fig. 5C, the activity of pmiR-RB-REPORT ${ }^{\mathrm{TM}}$-WT but not pmiR-RB-REPORT ${ }^{\mathrm{TM}}$-mut was significantly inhibited by miR-31a-5p mimics. Collectively, we argue that $\mathrm{Tp} 53$ is a direct target of miR-31a-5p.

\section{Discussion}

In the present study, we investigated the role of miR-31a-5p in apoptosis in AngII-stimulated H9C2 cells, a cardiac cell line.
We found that i) the miR-31a-5p level was decreased by AngII stimulation, ii) the Tp53 level was increased by AngII stimulation, and iii) Tp53 was a direct target of miR-31a-5p.

Previously, we showed that miR-31a-5p was significantly upregulated in the hearts of rats with end-stage HF compared to that in the sham-operation group. It is widely recognized that multi-factorial mechanisms are involved in the pathogenesis of HF, including increased activity of the RAAS and apoptosis. It is well documented that AngII is increased in pressure overload-induced cardiac hypertrophy (26), which contributes to cell hypertrophy, apoptosis, and fibroblast proliferation in vivo $(27,28)$ as well as myocardial cell apoptosis in vitro $(25)$. Indeed, ACEI has been used to treat HF effectively in the clinic, and the inhibition of ACE reduces cardiomyocyte apoptosis $(29,30)$, suggesting the implication of AngII signaling in cardiomyocyte apoptosis in vivo. Also, AngII is recognized as one of the triggers of G-protein-coupled receptor (GPCR) signaling pathways (31). In our study, miR-31a-5p negatively influenced AngII-induced cell apoptosis, suggesting that miR-31a-5p is involved in mediating GPCR signaling. In addition, since increased apoptosis of myocardial cells induces a loss of normal heart tissues and contributes to the pathogenesis and progression of cardiomyopathy (32), suppression of apoptosis is a potentially effective treatment for a number of cardiovascular diseases including HF (33). Consistent with the previous findings, in the present study, we found that AngII stimulation increased apoptosis and decreased miR-31a-5p expression in $\mathrm{H} 9 \mathrm{C} 2$ cells. Correspondingly, the increased expression of miR-31a-5p attenuated AngII-linked cell 
apoptosis, as evidenced by a decreased cell apoptotic rate and caspase-3 activity in AngII-treated cells with overexpressed miR-31a-5p, although miR-31a-5p only slightly reduced cell apoptosis in the absence of AngII. In contrast, the decreased expression of miR-31a-5p heightened AngII-induced cell apoptosis. Thus, it appears that miR-31a-5p is more involved in AngII-induced cardiomyocyte apoptosis than in the basal level of apoptosis. It will be interesting to explore whether miR-31a-5p is involved in other stress-induced myocardial cell apoptosis.

To further understand the molecular basis underlying miR-31a-5p mediation of cell apoptosis, we analyzed the expression profile of the apoptosis-related genes after miR-31a-5p overexpression in cells using a Rat Apoptosis RT $^{2}$ Profiler $^{\mathrm{TM}}$ PCR Array. We found that 16 proapoptotic genes were downregulated and 4 were upregulated, including the members of the caspase family and the $\mathrm{Bcl} 2$ family. The caspase family proteases are well known to serve important roles in initiation and development of apoptosis, which are influenced by either the extrinsic or intrinsic cues. Among these caspase family members detected, caspases 8 and 9 are regarded as initiators to receive signals from upstream, and caspases 3,6 , and 7 are death executors $(1,34,35)$. The Bcl 2 family including anti/proapoptotic genes is involved in the intrinsic apoptosis pathway; they are localized in the outer membrane of mitochondria and govern mitochondrial perforation or caspase recruitment (36). Thus, it appears that miR-31a-5p mediates AngII-induced apoptosis through affecting the expression of a number of apoptosis-related genes.

In the present study, we also identified a novel target of miR-31a-5p, Tp53. Both Tp53, the most frequently mutated gene in cancers, and miRNAs are implicated in a variety of cellular events and disease development, and the functional interaction between Tp53 signaling and miRNAs has also been explored previously $(37,38)$. For instance, Tp53 has been shown to govern the expression of miR-34 family members, which in turn potentiate Tp53-regulated apoptosis $(39,40)$. Also, Tp53 and its signaling are mediated by miRNAs. For example, miR-504 has been reported to suppress Tp53 expression once ectopically expressed, thus impairing Tp53-mediated apoptosis (41). In addition, miR-125b, a brain-enriched miRNA, negatively regulates the expression of Tp53 (42). Moreover, overexpression of miR-125b has been shown to diminish the expression level of Tp53 and promote cell apoptosis, while a decreased expression of miR-125b did the opposite (42). In the present study, we found that both miR-31a-5p and Tp53 were mediated by AngII stimulation. Furthermore, we observed an increased expression of Tp53, which coincided with a decreased expression of miR-31a-5p. Further examination using bioinformatics tools revealed that the 3'UTR of Tp53 mRNA contains the specific (i.e., seed) sequence for potential binding of miR-31a-5p. We further confirmed that Tp53 was a novel direct target of miR-31a-5p, as revealed by the findings that miR-31a-5p suppressed the activity of pmiR-RB-REPORT $^{\mathrm{TM}}$-WT but not pmiR-RB-REPORT ${ }^{\mathrm{TM}}$-mut. Together, these results suggested that pmiR-RB-REPORT ${ }^{\mathrm{TM}}-\mathrm{WT}$ bound to the seed sequence of the 3'UTR of TP53 mRNA and downregulated its expression.

In conclusion, our research demonstrated that the miR-31a-5p level is suppressed under AngII stimulation and that overexpression of miR-31a-5p reduces AngII-induced apoptosis in H9C2 cells. We further revealed that miR-31a-5p mediates AngII-triggered apoptosis at least in part through directly targeting Tp53 to its 3'UTR of mRNA. Therefore, our findings add another layer of complexity to the functional interaction between Tp53 and miRNAs. Further work should be directed at examining whether miR-31a-5p is a general regulator of apoptosis in response to different external stressors in the cardiovascular setting.

\section{Acknowledgements}

The present study was supported by National Natural Science Foundation of China (grant no. 81570360).

\section{References}

1. Salvesen GS and Dixit VM: Caspase activation: The induced-proximity model. Proc Natl Acad Sci USA 96: 10964-10967, 1999.

2. Narula J, Haider N, Virmani R, DiSalvo TG, Kolodgie FD, Hajjar RJ, Schmidt U, Semigran MJ, Dec GW and Khaw BA: Apoptosis in myocytes in end-stage heart failure. N Engl J Med 335: 1182-1189, 1996.

3. Kerr JF, Wyllie AH and Currie AR: Apoptosis: A basic biological phenomenon with wide-ranging implications in tissue kinetics. Br J Cancer 26: 239-257, 1972.

4. Colucci WS: Apoptosis in the heart. N Engl J Med 335: 1224-1226, 1996.

5. Wencker D, Chandra M, Nguyen K, Miao W, Garantziotis S, Factor SM, Shirani J, Armstrong RC and Kitsis RN: A mechanistic role for cardiac myocyte apoptosis in heart failure. J Clin Invest 111: 1497-1504, 2003.

6. Ma TK, Kam KK, Yan BP and Lam YY: Renin-angiotensin-aldosterone system blockade for cardiovascular diseases: Current status. Br J Pharmacol 160: 1273-1292, 2010.

7. Suzuki J, Iwai M, Nakagami H, Wu L, Chen R, Sugaya T, Hamada M, Hiwada $\mathrm{K}$ and Horiuchi $\mathrm{M}$ : Role of angiotensin II-regulated apoptosis through distinct AT1 and AT2 receptors in neointimal formation. Circulation 106: 847-853, 2002.

8. Demers C, Mody A, Teo KK and McKelvie RS: ACE inhibitors in heart failure: What more do we need to know? Am J Cardiovasc Drugs 5: 351-359, 2005.

9. Smith WH and Ball SG: ACE inhibitors in heart failure: An update. Basic Res Cardiol 95 (Suppl 1): I8-I14, 2000.

10. Zhao H, Qi G, Han Y, Shen X, Yao F, Xuan C, Gu Y, Qian SY, Zeng Q, O'Rourke ST, et al: 20-hydroxyeicosatetraenoic acid is a key mediator of angiotensin II-induced apoptosis in cardiac myocytes. J Cardiovasc Pharmacol 66: 86-95, 2015.

11. Schröder D, Heger J, Piper HM and Euler G: Angiotensin II stimulates apoptosis via TGF-betal signaling in ventricular cardiomyocytes of rat. J Mol Med (Berl) 84: 975-983, 2006.

12. Grishko V, Pastukh V, Solodushko V, Gillespie M, Azuma J and Schaffer S: Apoptotic cascade initiated by angiotensin II in neonatal cardiomyocytes: Role of DNA damage. Am J Physiol Heart Circ Physiol 285: H2364-H2372, 2003.

13. Cakmak HA, Coskunpinar E, Ikitimur B, Barman HA, Karadag B, Tiryakioglu NO, Kahraman K and Vural VA: The prognostic value of circulating microRNAs in heart failure: Preliminary results from a genome-wide expression study. J Cardiovasc Med (Hagerstown) 16: 431-437, 2015.

14. Cheng Y, Liu X, Zhang S, Lin Y, Yang J and Zhang C: MicroRNA-21 protects against the $\mathrm{H}(2) \mathrm{O}(2)$-induced injury on cardiac myocytes via its target gene PDCD4. J Mol Cell Cardiol 47: 5-14, 2009.

15. Flynt AS, Li N, Thatcher EJ, Solnica-Krezel L and Patton JG: Zebrafish miR-214 modulates Hedgehog signaling to specify muscle cell fate. Nat Genet 39: 259-263, 2007.

16. Lv G, Shao S, Dong H, Bian X, Yang X and Dong S: MicroRNA-214 protects cardiac myocytes against H2O2-induced injury. J Cell Biochem 115: 93-101, 2014.

17. Valastyan S, Chang A, Benaich N, Reinhardt F and Weinberg RA: Activation of miR-31 function in already-established metastases elicits metastatic regression. Genes Dev 25: 646-659, 2011. 
18. Pacurari $\mathrm{M}$ and Tchounwou PB: Role of MicroRNAs in renin-angiotensin-aldosterone system-mediated cardiovascular inflammation and remodeling. Int J Inflam 2015: 101527, 2015.

19. Rayner KJ: MicroRNA-155 in the heart: The right time at the right place in the right cell. Circulation 131: 1533-1535, 2015

20. Elton TS, Selemon H, Elton SM and Parinandi NL: Regulation of the MIR155 host gene in physiological and pathological processes. Gene 532: 1-12, 2013.

21. Jeppesen PL, Christensen GL, Schneider M, Nossent AY, Jensen HB, Andersen DC, Eskildsen T, Gammeltoft S, Hansen JL and Sheikh SP: Angiotensin II type 1 receptor signalling regulates microRNA differentially in cardiac fibroblasts and myocytes. Br J Pharmacol 164: 394-404, 2011.

22. Eskildsen TV, Schneider M, Sandberg MB, Skov V, Brønnum H, Thomassen M, Kruse TA, Andersen DC and Sheikh SP The microRNA-132/212 family fine-tunes multiple targets in Angiotensin II signalling in cardiac fibroblasts. J Renin Angiotensin Aldosterone Syst 16: 1288-1297, 2015.

23. Mlcochova J, Faltejskova-Vychytilova P, Ferracin M, Zagatti B, Radova L, Svoboda M, Nemecek R, John S, Kiss I, Vyzula R, et al: MicroRNA expression profiling identifies miR-31-5p/3p as associated with time to progression in wild-type RAS metastatic colorectal cancer treated with cetuximab. Oncotarget 6: 38695-38704, 2015

24. Liu X, Meng H, Jiang C, Yang S, Cui F and Yang P: Differential microRNA expression and regulation in the rat model of post-infarction heart failure. PLoS One 11: e0160920, 2016.

25. Cigola E, Kajstura J, Li B, Meggs LG and Anversa P: Angiotensin II activates programmed myocyte cell death in vitro. Exp Cell Res 231: 363-371, 1997

26. Kajstura J, Mansukhani M, Cheng W, Reiss K, Krajewski S Reed JC, Quaini F, Sonnenblick EH and Anversa P: Programmed cell death and expression of the protooncogene bcl-2 in myocytes during postnatal maturation of the heart. Exp Cell Res 219: $110-121,1995$.

27. Sadoshima J and Izumo S: Molecular characterization of angiotensin II-induced hypertrophy of cardiac myocytes and hyperplasia of cardiac fibroblasts. Critical role of the AT1 receptor subtype. Circ Res 73: 413-423, 1993.

28. Kajstura J, Cigola E, Malhotra A, Li P, Cheng W, Meggs LG and Anversa P: Angiotensin II induces apoptosis of adult ventricular myocytes in vitro. J Mol Cell Cardiol 29: 859-870, 1997.

29. Goussev A, Sharov VG, Shimoyama H, Tanimura M, Lesch M, Goldstein S and Sabbah HN: Effects of ACE inhibition on cardiomyocyte apoptosis in dogs with heart failure. Am J Physiol 275 H626-H631, 1998.
30. Bäcklund T, Palojoki E, Saraste A, Grönholm T, Eriksson A Lakkisto P, Vuolteenaho O, Nieminen MS, Voipio-Pulkki LM, Laine $\mathrm{M}$ and Tikkanen I: Effect of vasopeptidase inhibitor omapatrilat on cardiomyocyte apoptosis and ventricular remodeling in rat myocardial infarction. Cardiovasc Res 57: 727-737, 2003.

31. Liu CH, Gong Z, Liang ZL, Liu ZX, Yang F, Sun YJ, Ma ML, Wang YJ, Ji CR, Wang YH, et al: Arrestin-biased AT1R agonism induces acute catecholamine secretion through TRPC 3 coupling. Nat Commun 8: 14335, 2017.

32. Prech M, Marszałek A, Schröder J, Filas V, Lesiak M, Jemielity M, Araszkiewicz A and Grajek S: Apoptosis as a mechanism for the elimination of cardiomyocytes after acute myocardial infarction. Am J Cardiol 105: 1240-1245, 2010.

33. Kim NH and Kang PM: Apoptosis in cardiovascular diseases: Mechanism and clinical implications. Korean Circ J 40: 299-305, 2010.

34. Thornberry NA and Lazebnik Y: Caspases: Enemies within. Science 281: 1312-1316, 1998.

35. Sadowski-Debbing K, Coy JF, Mier W, Hug H and Los M Caspases-their role in apoptosis and other physiological processes as revealed by knock-out studies. Arch Immunol Ther Exp (Warsz) 50: 19-34, 2002.

36. Crow MT, Mani K, Nam YJ and Kitsis RN: The mitochondrial death pathway and cardiac myocyte apoptosis. Circ Res 95: 957-970, 2004

37. Hünten S, Siemens H, Kaller $M$ and Hermeking $H$ : The p53/microRNA network in cancer: Experimental and bioinformatics approaches. Adv Exp Med Biol 774: 77-101, 2013.

38. Feng Z, Zhang C, Wu R and $\mathrm{Hu} \mathrm{W}$ : Tumor suppressor p53 meets microRNAs. J Mol Cell Biol 3: 44-50, 2011.

39. Tazawa $H$, Tsuchiya $N$, Izumiya $M$ and Nakagama $H$ : Tumor-suppressive miR-34a induces senescence-like growth arrest through modulation of the E2F pathway in human colon cancer cells. Proc Natl Acad Sci USA 104: 15472-15477, 2007.

40. Raver-Shapira N, Marciano E, Meiri E, Spector Y, Rosenfeld N, Moskovits N, Bentwich Z and Oren M: Transcriptional activation of miR-34a contributes to p53-mediated apoptosis. Mol Cell 26: 731-743, 2007.

41. Hu W, Chan CS, Wu R, Zhang C, Sun Y, Song JS, Tang LH, Levine AJ and Feng Z: Negative regulation of tumor suppressor p53 by microRNA miR-504. Mol Cell 38: 689-699, 2010.

42. Le MT, Teh C, Shyh-Chang N, Xie H, Zhou B, Korzh V, Lodish HF and Lim B: MicroRNA-125b is a novel negative regulator of p53. Genes Dev 23: 862-876, 2009. 